\title{
Erratum: Developmental Isolation and Subsequent Adult Behavior of Drosophila paulistorum. III. Alternative Rearing ${ }^{1}$
}

\author{
Yong-Kyu Kim, ${ }^{2,4}$ H. Roberta Koepfer, ${ }^{3}$ and Lee Ehrman ${ }^{2}$
}

The following corrections should be noted in the above printed article:

P. 30, first column, line 21 , should read: "...index is $\left.\left[1-I^{2}\right) / N\right]^{1 / 2}$.

P. 30, second column, lines 34 and 35 , should read: "...i.e., naive $\mathrm{AN} q \times$ treated $\mathrm{TR} \delta$ and treated TR $+\times$ naive $\mathrm{AN} \delta$.

P. 31, Table I, line (B), third mating combination, should read: "TR $q \times$ AN $\delta$."

'This article appeared in Behav. Gen. 26(1):27-37 (1996).

2Division of Natural Sciences, State University of New York, Purchase, New York 10577. Fax: (914) 251-6635; e-mail: ykkim@purvid.purchase.edu.

${ }^{3}$ Biology Department, Queens College, Flushing, New York 11367.

${ }^{4}$ To whom correspondence should be addressed.
P. 32, first column, line 5, should read: “...CA $9 \times$ MN 0 matings." Line 7, should read: “...AN $9 \times$ CA $\delta^{\dagger} . "$

P. 33, Table III, last column, line 3, should read: " $0.57 \pm 0.08$. ."

P. 34, first column, line 14 , should read: “...(I $I_{\mathrm{CT} P}=0.53 \pm 0.11, I_{\mathrm{S} 9}=0.70 \ldots$.." Line 16 , should read: " $\ldots\left(I_{\mathrm{Cr} \rho}=0.93 \pm 0.05, I_{\mathrm{s} \rho}=0.43\right.$ $\pm 0.12 \ldots$

Line 36, second column, second Kim et al. reference, should read: Kim, Y.-K., Ehrman, L., and Koepfer, H.R. (1996). Developmental isolation and subsequent adult behavior of Drosophila paulistorum. II. Prior experience. Behav. Gen. 26:1525. 